\section{A safe and inexpensive method for setting up the Westergren ESR}

\section{A. K. RAY From the Department of Haematology, Royal Marsden Hospital, London}

A recently introduced disposable kit $^{1}$ for estimating the ESR has considerable advantages over previous methods since it avoids the hazard of pipetting by mouth. The Westergren pipette is introduced into a tightly fitting plastic tube containing citrated blood which then fills the pipette under pressure. There is no need to transfer it to a rack and the risk of spilling is eliminated. All the components of the kit are disposable.

A modification of this method is described which is considerably cheaper, although the kit is not entirely disposable. It ensures increased safety at a cost which works out at approximately 0.2 pence per test.

\section{Materials}

Conventional glass Westergren pipettes, stoppered plastic tubes, $63 \mathrm{~mm} \times 9 \mathrm{~mm}^{2}$, rubber test tube rack $^{3}$, and a $1 \mathrm{ml} \mathrm{DL}$ syringe pipette 4 .

\section{Method}

With a cork borer a hole is bored in the plastic stopper so that it forms a tight fit with a Westergren pipette pushed through it. A mark is made on the plastic tube to indicate a volume of $1.7 \mathrm{ml}$ (the minimum volume required to fill the Westergren pipette). This is easily done by placing the plastic tubes in batches into the holes of a wooden block of a suitable depth and rotating them against the tip of a marking pen. It is a simple process to maintain a stock of marked tubes and bored stoppers. Then $0.34 \mathrm{ml}$ of $3.8 \%$. sodium citrate solution is delivered into the marked tubes from a $1 \mathrm{ml} \mathrm{DL}$ syringe pipette set to deliver this volume. Well mixed blood from a sequestrene bottle is then simply poured into the tube up to the mark. The stopper is placed on the tip of the Westergren pipette and then placed firmly in position into the tube (see Figure, position 2). With a finger over the open end of the Westergren

\footnotetext{
${ }^{1}$ Dispettes, Guest Medical \& Dental Products AG, Switzerland ${ }^{2}$ LP/3 tubes, Luckham Ltd.

${ }^{3}$ Rubber holder, TTS 6, 56 holes $\frac{8}{8}$ in. dia. Esco (Rubber) Ltd. 'DL syringe pipette, Aimer Products Ltd.
}

Received for publication 30 September 1971.

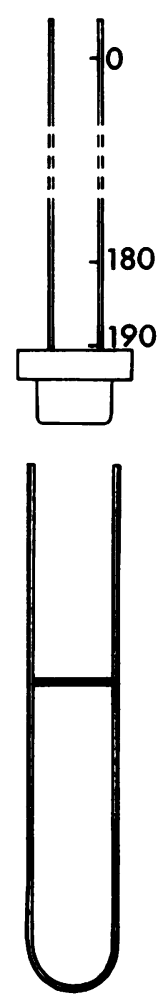

1

Fig. The method demonstrated

pipette the whole preparation is inverted several times for thorough mixing. The Westergren pipette is pushed gently into the tube until the blood/citrate mixture reaches the zero mark (Figure, position 3 ). The tube is then placed in the rubber holder.

At the end of the test the tube of blood is discarded and the Westergren tube with its stopper is dropped into a virus-neutralizing solution such as glutaraldehyde.

\title{
Conclusion
}

The stoppered tubes are reasonably priced and the stoppers may be used many times. The size of the tube compared with the total volume of blood allows ample space for thorough mixing. The glass Westergren pipette can be disinfected without losing its markings. Using the cone screw cap sequestrene bottles ${ }^{5}$ no blood comes in contact with the operator's skin.

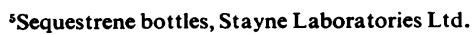

